\title{
Variable-period permanent magnet undulators
}

\author{
N. A. Vinokurov, ${ }^{1,2}$ O. A. Shevchenko, ${ }^{1}$ and V. G. Tcheskidov ${ }^{1}$ \\ ${ }^{1}$ Budker Institute of Nuclear Physics, 11 Academician Lavrentyev Prospect, 630090, Novosibirsk, Russia \\ ${ }^{2}$ Novosibirsk State University, 2 Pirogova, 630090, Novosibirsk, Russia
}

(Received 31 October 2010; published 1 April 2011)

\begin{abstract}
To change the wavelength of undulator radiation, variation of the undulator magnetic field amplitude is frequently applied. Another option is changing the undulator period. A corresponding scheme is described. Both the period and number of periods can be changed. For a set of undulator sections (as in the x-ray free-electron lasers ), the mechanical motion of the periods allows doing without phase shifters between the undulator sections. Magnetic field calculations for some undulator parameters of interest were performed. Numerous advantages of the new undulators (fixed gap, strong dependence of the undulator radiation wavelength on the period, relatively low field amplitude variation, and variable number of periods) look very attractive. The prospects for this new type of undulators are discussed.
\end{abstract}

PACS numbers: 07.85.Qe, 41.60.Cr

\section{INTRODUCTION}

Contemporary storage-ring-based $\mathrm{x}$-ray sources and free-electron lasers use magnetic undulators. Undulators, or wigglers, are dedicated magnetic systems which create a spatially periodic transverse magnetic field. A profound description of different types of undulators can be found in $[1,2]$. For most of the applications, it is necessary to tune the wavelength of the undulator radiation in the forward direction,

$$
\lambda=\lambda_{u} \frac{1+K^{2}}{2 \gamma^{2}},
$$

where $\lambda_{u}$ is the undulator period, and $\gamma$ is the electron energy divided by its rest energy $m c^{2}$. The undulator parameter $K$ takes into account the reduction in the longitudinal velocity of an electron due to the curvature of the wiggling trajectory. For a planar undulator with a sinusoidal longitudinal dependence of the vertical magnetic field, $K=e B_{0} \lambda_{u} /\left(2 \sqrt{2} \pi m c^{2}\right)$ and $B_{0}$ is the field amplitude.

Typically, variation of particle energy is impossible (in a storage ring with a lot of undulators) or complicated (because of focusing and trajectory change). A frequent way of tuning the undulator radiation wavelength is varying the field amplitude $B_{0}$. In electromagnetic undulators it is realized via varying current in the coils, and in permanent magnet undulators, mainly varying the undulator gap. Serious intrinsic drawbacks of this technique are too low field values at short wavelengths and too high ones at long wavelengths. This circumstance is, in particular, an obstacle for using undulators with a very short period, because the tuning range of such undulators is very small as

Published by American Physical Society under the terms of the Creative Commons Attribution 3.0 License. Further distribution of this work must maintain attribution to the author(s) and the published article's title, journal citation, and DOI. the $K$ parameter is proportional to the undulator period. Tuning wavelength via varying the field amplitude is especially difficult in the x-ray free-electron lasers (FELs) [3]. A decrease in the field amplitude in these devices may cause a significant increase in the gain length. Moreover, the field amplitude must be the same in different sections of a long undulator, with a precision of better than $0.1 \%$. Tuning the gaps of all undulator sections with such precision is a significant technological challenge.

Another solution for wavelength tuning is the variation of the undulator period $\lambda_{u}$. The "simplest" way to do it is to replace one undulator with another [4,5]. In such a design, fine wavelength tuning can be realized via gap variation. Different electromagnetic variable-period undulators (VPU) were discussed in papers [6-8].

\section{SPLIT-POLE UNDULATOR}

Let us consider first the hybrid permanent magnet undulator (PMU) $[9,10]$. We propose dividing all poles (iron or permendure) into two halves, as shown in Fig. 1.

It is easy to estimate the magnetic field amplitude $B_{0}$ in this undulator. In rather high magnetic blocks, magnetic induction is almost zero and magnetic field intensity is close to the coercivity $H_{c}$. Then the scalar magnetic potential of a pole is $\pm H_{c} t / 2$ ( $t$ is the thickness of the permanent magnet, see Fig. 1). For a minimum period, when the undulator is the conventional hybrid undulator, a plot of the scalar magnetic potential at the gap boundary $y=g / 2$ ( $g$ is the value of the undulator gap) is shown in Fig. 2. To draw it, one has to connect the ends of the straight-line segments, which correspond to the undulator poles, using segments of some curve.

To find the first harmonics of the potential, one can approximate this curve with a trapezoidal one. Then the amplitude of the first harmonics of the potential is 

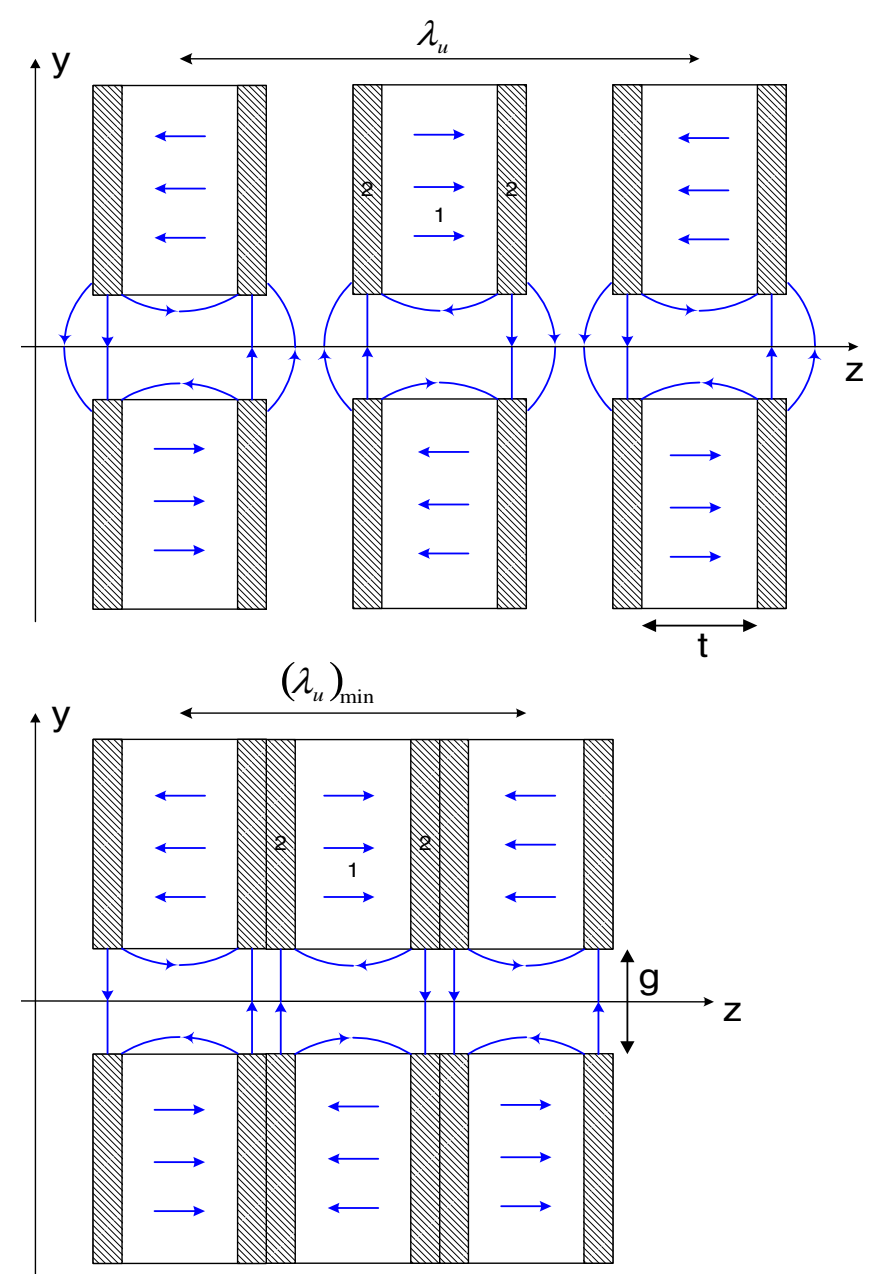

FIG. 1. The hybrid permanent magnet undulator with split poles (side view). (1) Magnet blocks. (2) Iron (or permendure). Lines of magnetic induction are shown with arrows. The lower scheme represents the minimum period, when the split-pole undulator becomes a conventional hybrid undulator.

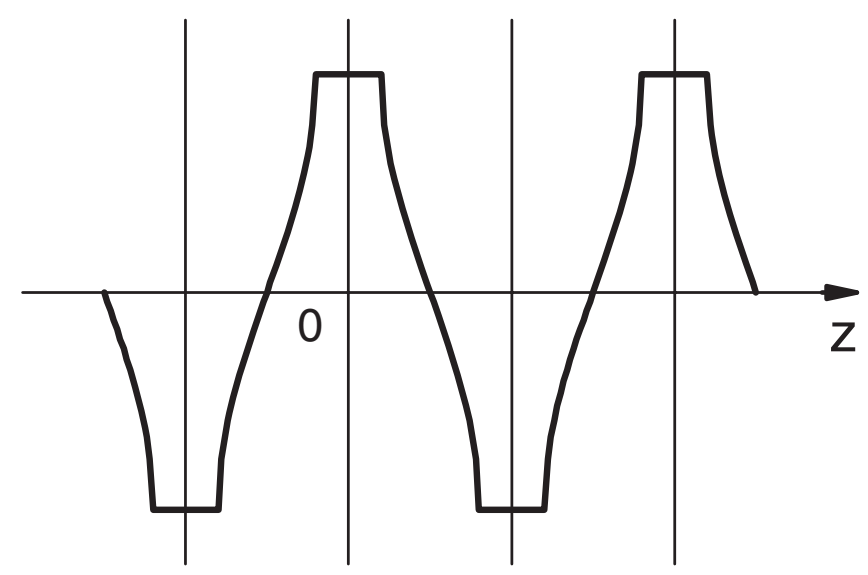

FIG. 2. Potential at the upper boundary.

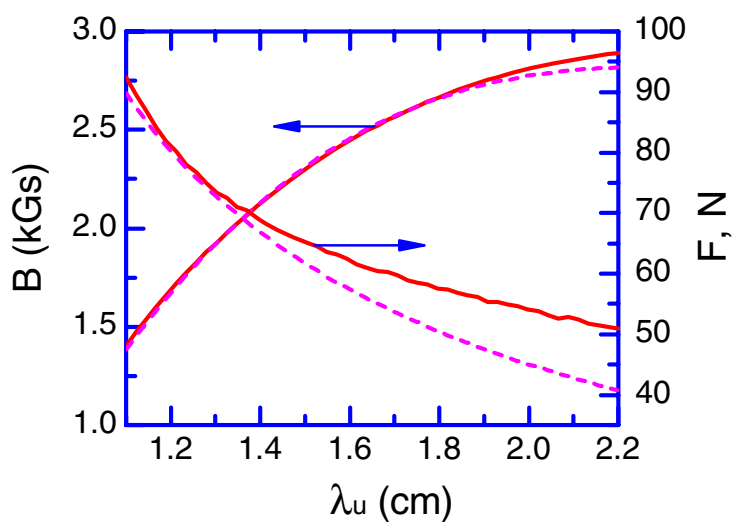

FIG. 3. Dependence of the first harmonic amplitude and repulsion force on the undulator period. The solid curves are for the simulation results, the dashed curves are for the analytical expressions.

$$
B_{0} \frac{\lambda_{u}}{2 \pi} \sinh \frac{\pi g}{\lambda_{u}}=H_{c} \frac{2 \lambda_{u}}{\pi^{2}} \sin \frac{\pi t}{\lambda_{u}} .
$$

Equation (2) leads to a simple estimate of the field amplitude:

$$
B_{0}=\frac{4}{\pi} H_{c} \frac{\sin \frac{\pi t}{\lambda_{u}}}{\sinh \frac{\pi g}{\lambda_{u}}} \rightarrow H_{c} \frac{\sin \frac{\pi t}{\lambda_{u}}}{\sinh \frac{\pi g}{\lambda_{u}}} .
$$

Because of the finite height (and width) of the magnetic blocks, the magnetic field intensity in them is less than $H_{c}$. That allows us to replace the factor $4 / \pi$ in Eq. (3) with unity. To take into account the breaks between the halves of the undulator poles, which appear when the period is increased, one has to multiply this amplitude by the factor

$$
\kappa=1-\frac{\pi}{\tanh \left(\frac{\pi g}{\lambda_{u}}\right)} \frac{1}{\sin c\left(\frac{\pi t}{\lambda_{u}}\right)}\left(\frac{\Delta}{\lambda_{u}}\right)^{2},
$$

where $\Delta$ is the break length.

The modified Eq. (3) gives a very good approximation for a general case with an increased period, as demonstrated in Fig. 3. The numerical calculations were

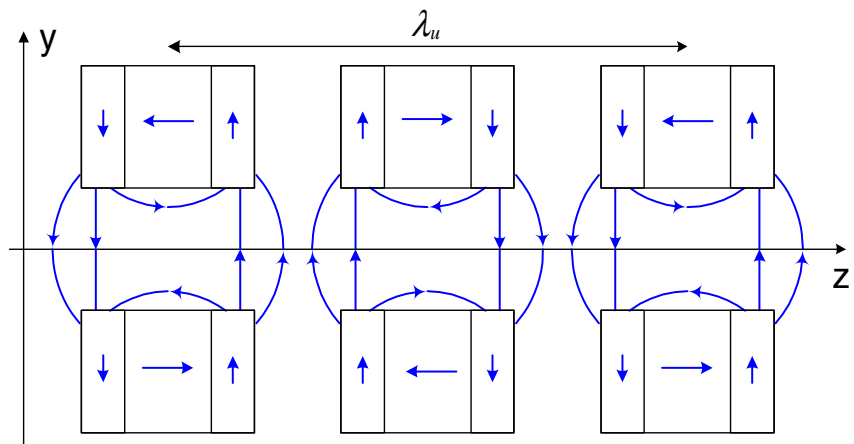

FIG. 4. The pure permanent magnet undulator with split "poles" (side view). Lines of magnetic induction are shown with arrows. 
performed with the 2D code FEMM 3.4[11] and used the following values: a pole height of $1 \mathrm{~cm}$, a magnet height of $1.3 \mathrm{~cm}, H_{c}=13 \mathrm{kOe}, g=1 \mathrm{~cm}, t=0.41 \mathrm{~cm}$.

A similar consideration may be applied to the pure permanent magnet undulator shown in Fig. 4.

\section{MECHANICAL DESIGN}

As shown in Fig. 1, the two half poles and permanent magnet block are assembled into a rigid unit. An interesting feature of this design is the strong longitudinal repulsion between the units. To estimate it, one can calculate the magnetic field pressure in the $x-y$ plane in the middle of a split pole. The field there has no longitudinal ( $z$ ) component, and it is maximal near the edges (top, bottom, left, and right) of the pole halves. A rough estimate of the repulsion force for a pole of a perimeter $P$ is

$$
F=\frac{H_{c}^{2}}{8 \pi} \frac{\left(\lambda_{u}\right)_{\min } P}{2 \pi^{2}} \arcsin \left(\frac{2 t}{\lambda_{u}}\right) .
$$

Comparison of this expression with the simulation results is presented in Fig. 3.

For a short-period undulator, it is of the order of $0.1 \mathrm{kN}$. Moreover, forces between different undulator half periods are equal with high accuracy. Therefore, fixing only the end units would be enough.

This feature opens the possibility of varying the number of periods during wavelength tuning. An example of such a design is shown in Fig. 5.

When the undulator period is increased, the pushers move the extra blocks to the side cartridge. The "feeders" may be placed near the undulator ends. Thus, the number of periods can also be variable, and the full length allocated

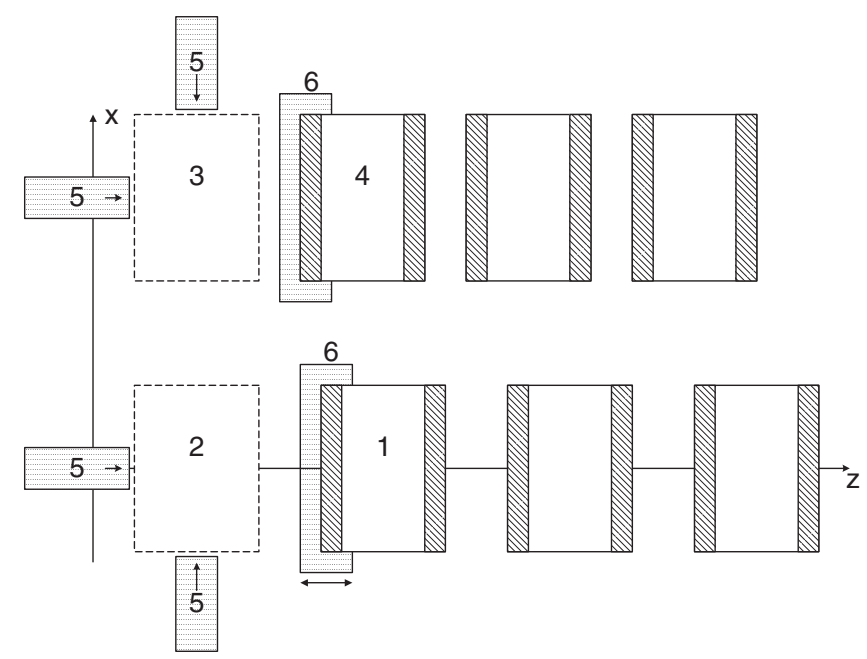

FIG. 5. One of the possible schemes of variation of the number of periods (top view). (1)-(4) Successive positions of the block being removed, (5) pushers, and (6) adjustable stops. The lower blocks are in the undulator, the upper ones in the cartridge for spare blocks. for the undulator is used. The longitudinal displacement of the undulator ends provides the possibility of phasing undulators in long undulator chains (as it takes place in a high-gain $\mathrm{x}$-ray FEL) without dedicated phase shifters.

The compensated terminations in this design are rather simple. Indeed, each half-period block provides zero first integral of the vertical field (trajectory kick). Therefore one weaker half period at either end of the undulator is enough to compensate the second integral (trajectory parallel shift) at this end. This compensation is perfect for the pure PMU shown in Fig. 4 in the approximation of the unit permeability. For the hybrid PMU, small residual decompensation is possible and has to be corrected via varying the shapes or with steering coils.

\section{THE LONGITUDINAL DISPLACEMENT TOLERANCES}

As the PMU half-period blocks move along the undulator axis, it is necessary to estimate the tolerances for their longitudinal positioning. Let us consider a small longitudinal shift $\Delta z \ll \lambda_{u}$ of one half-period block of the pure PMU, shown in Fig. 4. Certainly, such a shift does not lead to a transverse displacement of the trajectory at a long distance from the shifted block. It produces only a small trajectory bump, centered in the middle of the block center. It is easy to find the expression for the corresponding angle deviation - it would be enough to consider an undulator with infinitely wide and infinitely high magnet blocks, which gives us the upper estimate,

$$
\begin{aligned}
\frac{d x}{d z}= & \frac{H_{c} \Delta z}{2 \pi \gamma m c^{2}}\left[\frac{1}{2} \ln \frac{1+(\zeta-t / g)^{2}}{1+(\zeta+t / g)^{2}}+\operatorname{atan}(\zeta+2 t / g)\right. \\
& -\operatorname{atan}(\zeta+t / g)-\operatorname{atan}(\zeta-t / g)+\operatorname{atan}(\zeta-2 t / g)],
\end{aligned}
$$

where $\zeta=2 z / g$. The bump causes the delay of electron $c \Delta t=\int_{-\infty}^{\infty}(d x / d z)^{2} d z / 2$. It corresponds to the phase shift of the first harmonics of the undulator radiation,

$$
\begin{aligned}
\Delta \varphi & =\frac{2 \pi c \Delta t}{\lambda}=\left(\frac{H_{c} \Delta z}{m c^{2}}\right)^{2} \frac{t^{2}}{g \lambda_{u}\left(1+K^{2}\right)} G\left(\frac{t}{g}\right), \\
G(x)= & \frac{1}{4 \pi x^{2}} \int_{-\infty}^{\infty}\left[\frac{1}{2} \ln \frac{1+(\zeta-x)^{2}}{1+(\zeta+x)^{2}}+\operatorname{atan}(\zeta+2 x)\right. \\
& -\operatorname{atan}(\zeta+x)-\operatorname{atan}(\zeta-x) \\
& +\operatorname{atan}(\zeta-2 x)]^{2} d \zeta \approx 1 .
\end{aligned}
$$

If the shifted block is in the middle of the undulator, it leads to the $1-(\Delta \varphi)^{2} / 8$ reduction in the Fourier harmonics of the radiation field, but the maximum of the harmonics (which will be shifted) will be reduced by a factor of $1-(\Delta \varphi)^{2} / 32$ only. Taking into account that $t<\lambda_{u} / 4$, one obtains the simple limitation, 


$$
\Delta z<\frac{m c^{2}}{H_{c}} \sqrt{16 g\left(1+K^{2}\right)(\Delta \varphi)_{\max } / \lambda_{u}} .
$$

This value is about $1 \mathrm{~mm}$ for typical undulator parameters. For example, for $H_{c}=13 \mathrm{kOe}, K=1, \lambda_{u} / g=4$, and $(\Delta \varphi)_{\max }=0.1$, Eq. (8) gives $\Delta z<1.2 \mathrm{~mm}$.

The numerical calculations for the hybrid VPU shows that the total angular deflection caused by the shift of one half-period block is very small for rather wide magnets. Therefore, the longitudinal shift tolerances for them are almost the same as for the pure permanent magnet VPU.

For a more precise estimate of the undulator performance reduction, one can use the coupling factor $[2,12]$

$$
\begin{aligned}
F(L)= & \int_{0}^{L} I_{1 y}(z) \exp \left\{i \kappa _ { 0 } \left[z+\int_{0}^{z} I_{1 x}^{2}\left(z^{\prime}\right) d z^{\prime}\right.\right. \\
& \left.\left.+\int_{0}^{z} I_{1 y}^{2}\left(z^{\prime}\right) d z^{\prime}\right]\right\} d z,
\end{aligned}
$$

where

$$
\begin{aligned}
& I_{1 y}(z)=\frac{e}{m c^{2}} \int_{0}^{z} B_{y}\left(0,0, z^{\prime}\right) d z^{\prime} \\
& I_{1 x}(z)=\frac{e}{m c^{2}} \int_{0}^{z} B_{x}\left(0,0, z^{\prime}\right) d z^{\prime}
\end{aligned}
$$

and $\kappa_{0}=k /\left(2 \gamma^{2}\right)$. It describes the spontaneous emission (far zone radiation field amplitude, see, e.g., [13]) in the direction of the $z$ axis from the whole undulator length $L$. This value can be calculated from the magnetic measurements data and then compared with the ideal value. Therefore, it is a convenient way to specify the tolerances by the trajectory straightness (maximum trajectory deviation at a given energy) and maximum relative spontaneous radiation amplitude reduction $\Delta|F| /|F|_{\text {ideal }}$.

On the other hand, the coupling in Eq. (9) is the sum (integral) of the contributions of different parts of the undulator. Therefore, for a nonideal field it would be interesting to find the places where the relative phases of different contributions change. To see this, one can use the hodograph of $F(z)$. The hodograph of a complex function $F(z)$ is defined as the map of the $z$ axis into the plane $X=$ $\operatorname{Re} F(z), Y=\operatorname{Im} F(z)$ [14]. For a perfect undulator, the hodograph is a straight-line segment. For an imperfect undulator, it is a curve segment of the same length, but the distance between the beginning and the end, $|F(L)|$, is less than for the ideal case. This hodograph method is very similar to the usage of the Cornu spiral to describe the Fresnel diffraction [15]. It is very natural as $F$ is proportional to the emitted field amplitude. Each small segment of the hodograph is the contribution of some short segment of the undulator to the radiation field amplitude. For example, the localized phase shift of Eq. (6) corresponds to the jog of the hodograph by this angle.

Let us consider shifts of all blocks $\Delta z(z)$. If upper and lower blocks are shifted differently, we have to take a simple average of these shifts. As the phase error from the shift of one block of Eq. (6) is rather small, we will be interested in slow variations of $\Delta z(z)$, which give accumulating contributions to the phase shift and the coupling factor reduction. Then the undulator field can be expressed as

$$
\begin{aligned}
& B_{y}=\left(B_{0}+\frac{d B_{0}}{d \lambda_{u}} \frac{d \Delta z}{d z}\right) \cos \left[\frac{2 \pi(z-\Delta z)}{\lambda_{u}}+\varphi_{0}\right] . \\
& \text { Then, for } \kappa_{0}=2 \pi / \lambda_{u}\left(1+K^{2}\right), \\
& |F(L)| \\
& \approx \frac{K}{\sqrt{2}}\left|\int_{0}^{L} \exp \left\{\frac{2 \pi i \Delta z}{\lambda_{u}}\left[1+\frac{2 K^{2}}{1+K^{2}}\left(1+\frac{d \ln B_{0}}{d \ln \lambda_{u}}\right)\right]\right\} d z\right| .
\end{aligned}
$$

Expansion of Eq. (12) gives the simple reduction factor for the coupling

$$
\begin{aligned}
\left|\frac{F(L)}{F_{0}(L)}\right| \approx & 1-\left(\frac{2 \pi}{\lambda_{u}}\right)^{2}\left[1+\frac{2 K^{2}}{1+K^{2}}\left(1+\frac{d \ln B_{0}}{d \ln \lambda_{u}}\right)\right]^{2} \\
& \times \frac{\left\langle\Delta z^{2}\right\rangle-\langle\Delta z\rangle^{2}}{2},
\end{aligned}
$$

where $\langle\Delta z\rangle$ is the displacement averaged over the undulator length. For example, the dependence $\Delta z(z)$ caused by the friction lag is $a z^{2}+b z$, where $a$ is proportional to the friction constant, and $b$ may be adjusted by the motion of the end blocks. To minimize the displacement variance, one can choose $b=-a L$, which corresponds to the zero displacement at the undulator ends. Then the variance is $4\left(\Delta z_{\max }\right)^{2} / 45$. For $\Delta z_{\max }<\lambda_{u} / 20 \sim 1 \mathrm{~mm}$, the coupling reduction given by Eq. (13) is rather small.

Thus, one can conclude that the submillimeter precision of the longitudinal half-period positioning is enough for good quality of the VPU.

Another type of field errors caused by the longitudinal displacement of one half-period block is the nonzero longitudinal field $B_{z}$. It is clear that its integral is zero. Therefore, it causes neither kick nor betatron coupling.

The compensation of magnetization errors in the VPU is similar to that for other types of permanent magnet undulators. Speaking of the VPU, such compensation can be performed for each half-period block. Then, during magnetic measurements of the undulator, bad blocks may be just replaced with good ones.

\section{ADVANTAGES OF VARIABLE-PERIOD UNDULATORS}

To compare the VPU with the variable-amplitude undulator, we have to consider particular applications. The simplest one is the generation of spontaneous radiation. As for a conventional undulator, a significant $K$ value is required for wavelength tuning. Indeed, according to Eq. (1), one needs $K>1$ to increase the wavelength twice. Taking into account the field estimate of Eq. (3) and assuming $t=\lambda_{u} / 3$, we get 


$$
\frac{e H_{c} \lambda_{u} \sqrt{3}}{4 \sqrt{2} \pi m c^{2} \sinh \frac{\pi g}{\lambda_{u}}}>1 .
$$

For example, at $H_{c}=13 \mathrm{kOe}$ and $g=1 \mathrm{~cm}$ it gives $\lambda_{u}>2.4 \mathrm{~cm}$.

For the VPU, one can allow $K=0.1$ and have $\left(\lambda_{u}\right)_{\min }=$ $1.1 \mathrm{~cm}$ for the same parameters. Equations (3) and $\left(3^{\prime}\right)$ show that this period has to be increased up to $\lambda_{u}=2 \mathrm{~cm}$ to double the wavelength. For this period, $K=0.4$.

If we discuss the same wavelengths for these two undulators, the required particle energies differ by a factor of 1.45. For storage rings such an energy decrease is very significant. It reduces the beam emittance and synchrotron radiation losses and therefore the rf power. Moreover, low- $K$ undulators cause low tune shift, because the latter is proportional to the square of the magnetic field amplitude.

As for the higher harmonics of the undulator radiation, the spectral intensity dependencies on the wavelength for the VPU are weaker than for the variable-gap undulator. The results of corresponding calculations for an electron energy of $7 \mathrm{GeV}$ are shown in Figs. 6-10. A minimum gap value of $1 \mathrm{~cm}$ was used for these calculations. It is a typical value for storage rings. Certainly, there can be smaller gaps in the in-vacuum undulators, but these undulators have intrinsic problems with degradation of magnets, significant impedances, and decrease in the dynamic aperture. The magnetic field amplitude in these calculations was obtained from Eq. (3) with $H_{c}=10 \mathrm{kOe}$ and $t=1 \mathrm{~cm}$. In the VPU, the magnet blocks were of half thickness. The calculations were made for a constant VPU length, so the number of its periods was variable.

Another promising application is the high-gain x-ray FEL. As the required beam peak current in such a FEL is

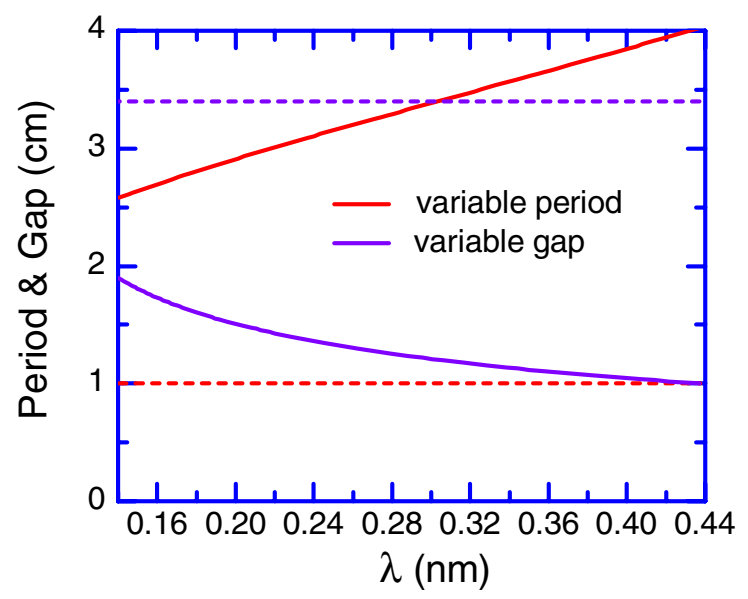

FIG. 6. The period of the VPU and the gap of the variable-gap undulator (solid lines) required to reach the given fundamental wavelength. The red dashed line is the constant gap of the VPU, and the blue dashed line is the constant period of the variablegap undulator.

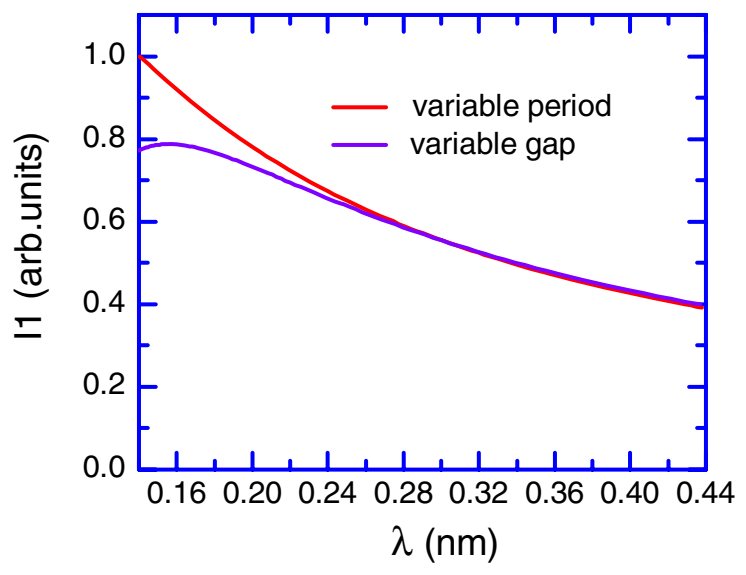

FIG. 7. The spectral intensities of the fundamental harmonics.

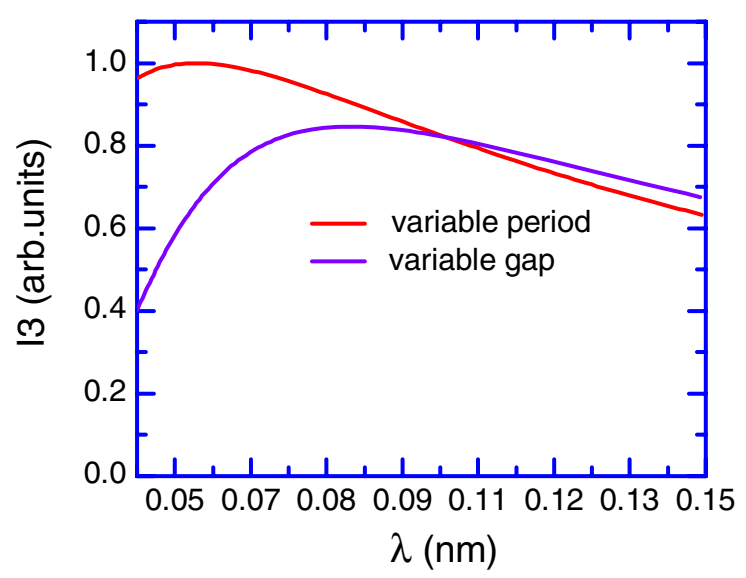

FIG. 8. The spectral intensities of the third harmonics.

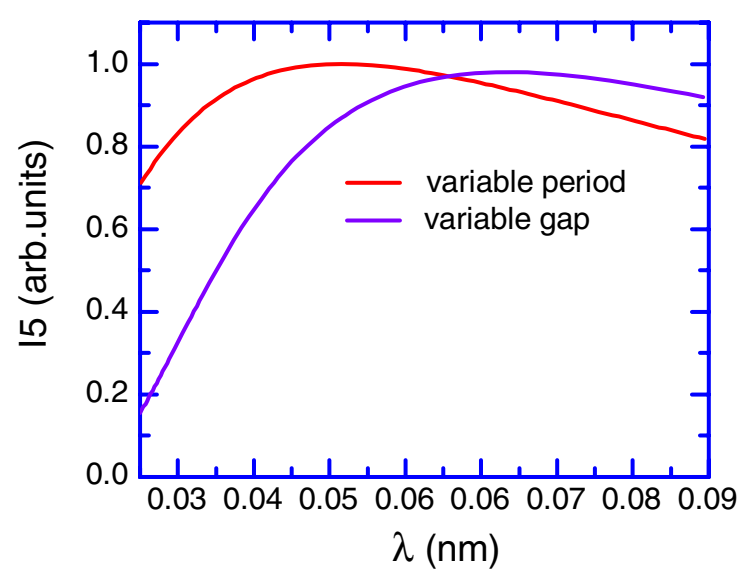

FIG. 9. The spectral intensities of the fifth harmonics.

rather high, the gap of the undulator is limited by wakefields (see, e.g., [16]). If the wavelength of such a FEL is changed via raising the undulator gap from its minimum value, the gain length increases significantly. The reason is not only the decrease in the particle-wave interaction due to a lower $K$ but the decrease in the wavelength and, 


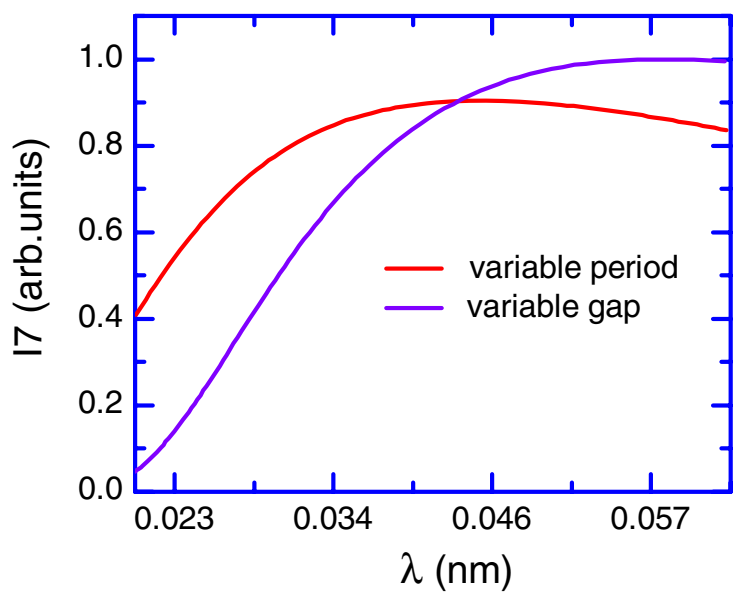

FIG. 10. The spectral intensities of the seventh harmonics.

correspondingly, the ratio of the wavelength to the beam emittance. If this ratio is small, a finite longitudinal velocity spread and transverse beam sizes lead to a significant increase in the gain length.

Another problem of the variable-gap undulators is the extremely tight tolerances for gap difference in different sections of the long undulators.

The VPU, on the contrary, can be optimized at the minimum wavelength and minimum gap. Then, at the shortest wavelength, the undulator gap (and therefore the period) is less than the maximum gap of the variablegap undulator. Therefore, the use of the VPU decreases the gain length and required beam energy of high-gain FELs. An example of the gain length dependence on the radiation wavelength is shown in Fig. 11.

The calculations were performed with the Ming Xie formula [17] for a minimum gap of $6 \mathrm{~mm}$, electron energy of $5.8 \mathrm{GeV}$, peak current of $2 \mathrm{kA}$, relative energy dispersion of $10^{-4}$, and normalized emittance of $0.2 \mu \mathrm{m}$. At the left edge of Fig. 11, the VPU period is the minimum $1.6 \mathrm{~cm}$ and the variable-gap undulator period is more than $6 \mathrm{~mm}$. At

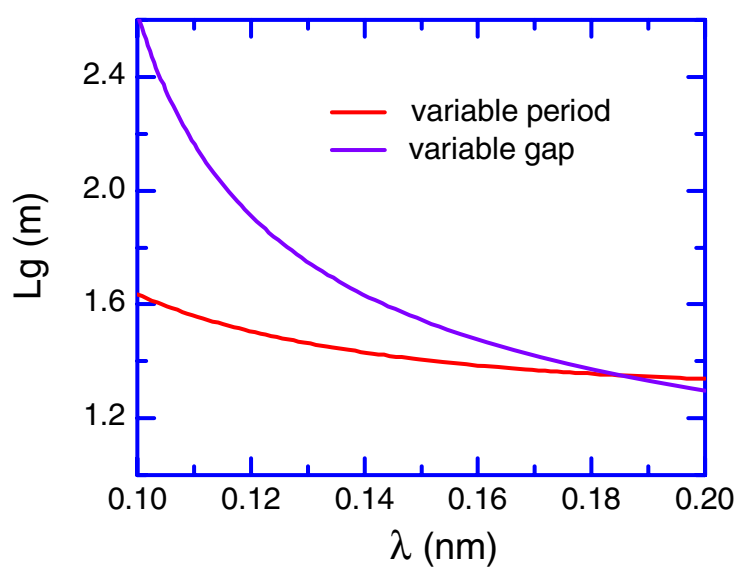

FIG. 11. The dependence of the gain length on the radiation wavelength.

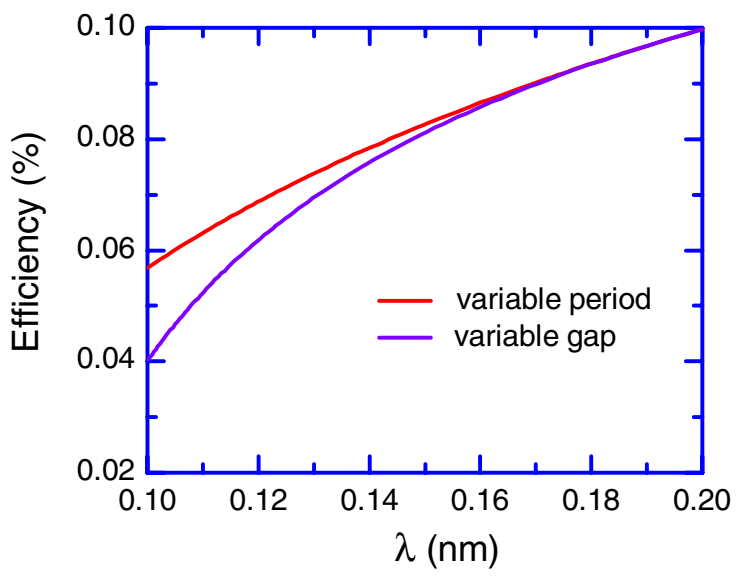

FIG. 12. The dependence of the FEL efficiency on the radiation wavelength.

the right edge, the gap of the variable-gap undulator is $6 \mathrm{~mm}$ and the period of the VPU is $2.14 \mathrm{~cm}$. A similar plot for the FEL efficiency (without tapering) is presented in Fig. 12.

Another advantage of the VPU is the possibility of using a precisely fixed gap (see Fig. 13). As it was discussed above, the tolerances for the period variations along the undulator are not so tight. Moreover, as it was mentioned already, the phase shifters may be eliminated.

Further performance improvement of the VPU is possible with the use of cooling to low temperatures [18]. This option is rather easy due to the small transverse sizes of the VPU.

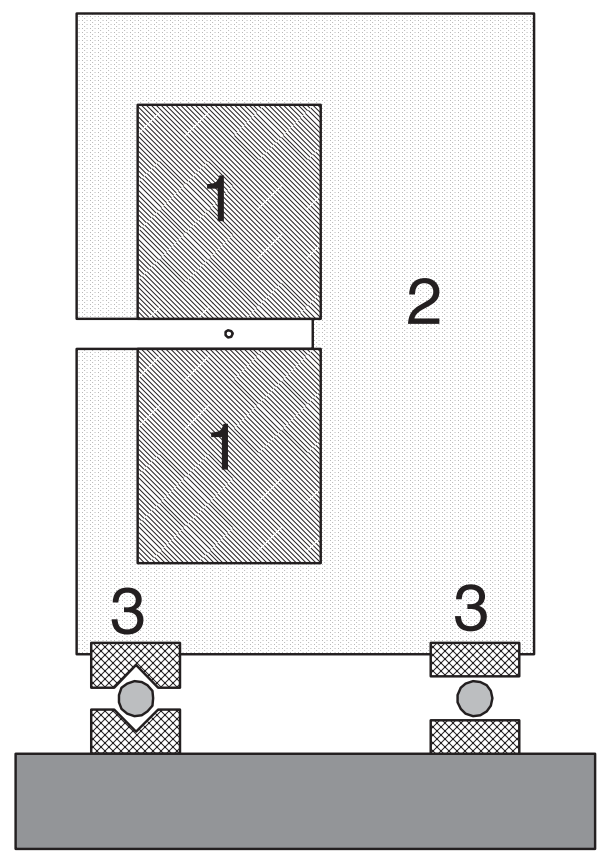

FIG. 13. Side view of a half period of the fixed-gap VPU. (1) Poles, (2) plate, and (3) ball-bearing guideways. 


\section{CONCLUSION}

A new design of permanent magnet undulators, the VPU, was considered in the paper. It opens new prospects for further improvements of accelerator-based radiation sources. The new design is especially attractive for the long undulator systems of the high-gain x-ray FELs. Certainly, like most new proposals, it has to be developed further in more detail. In particular, the mechanical design, which is a crucial part for the VPU, has to be done and proofed with a prototype.

\section{ACKNOWLEDGMENTS}

We would like to thank Dr. A. Zholents (ANL) for very useful discussions of the possible application of the VPU to the generation of high-order harmonics.

[1] J. A. Clarke, The Science and Technology of Undulators and Wigglers (Oxford University Press, New York, 2004).

[2] E. Levichev and N. Vinokurov, Rev. Accel. Sci. Technol. 3, 203 (2010).

[3] T. Tanaka, in Proceedings of the 31st International Free Electron Laser Conference (FEL 09), Liverpool, UK (STFC Daresbury Laboratory, Warrington, 2009), p. 524.

[4] R. Z. Bachrach et al., Proc. SPIE Int. Soc. Opt. Eng. 582, 251 (1985).
[5] G. Isoyama et al., Rev. Sci. Instrum. 60, 1863 (1989).

[6] A. van Steenbergen et al., in Proceedings of the IEEE 1991 Particle Accelerator Conference (APS Beams Physics) (IEEE, Piscataway NJ, 1991), p. 2724.

[7] G. K. Shenoy et al., J. Synchrotron Radiat. 10, 205 (2003).

[8] A. Bernhard et al., in Proceedings of the 11th European Particle Accelerator Conference, Genoa, 2008 (EPS-AG, Genoa, Italy, 2008), p. 2231.

[9] G. A. Kornyukhin et al., Nucl. Instrum. Methods 208, 189 (1983).

[10] E. Hoyer et al., Nucl. Instrum. Methods 208, 117 (1983).

[11] http://www.femm.info/wiki/HomePage.

[12] E. S. Gluskin et al., Nucl. Instrum. Methods Phys. Res., Sect. A 475, 323 (2001).

[13] L. D. Landau and E. M. Lifshits, The Classical Theory of Fields (Pergamon Press, Oxford, 1987).

[14] M. A. Lavrentyev and B. V. Shabat, Methods of the Theory of Functions of a Complex Variable (Fizmatgiz, Moscow, 1958) (in Russian).

[15] M. Born and E. Wolf, Principles of Optics (Pergamon Press, Oxford, 1968).

[16] K. Bane and G. Stupakov, in Proceedings of the 21st Particle Accelerator Conference, Knoxville, 2005 (IEEE, Piscataway, NJ, 2005), p. 3390, http://accelconf.web .cern.ch/AccelConf/p05/PAPERS/RPPE057.PDF.

[17] M. Xie, Nucl. Instrum. Methods Phys. Res., Sect. A 445, 59 (2000).

[18] T. Hara et al., Phys. Rev. ST Accel. Beams 7, 050702 (2004). 